\title{
One-stage Revision With Catheter Infusion of Intraarticular Antibiotics Successfully Treats Infected THA
}

\author{
Leo A. Whiteside MD, M. E. Roy PhD
}

Published online: 10 August 2016

(C) The Association of Bone and Joint Surgeons (B) 2016

\begin{abstract}
Background Two-stage revision surgery for infected total hip arthroplasty (THA) is commonly advocated, but substantial morbidity and expense are associated with this technique. In certain cases of infected THA, treatment with one-stage revision surgery and intraarticular infusion of antibiotics may offer a reasonable alternative with the distinct advantage of providing a means of delivering the drug in high concentrations.

Questions/purposes We describe a protocol for intraarticular delivery of antibiotics to the hip through an indwelling catheter combined with one-stage revision surgery and examine (1) the success as judged by eradication of infection at 1 year when treating chronically infected cemented stems; (2) success in treating late-onset acute
\end{abstract}

One of the authors (LAW) certifies that he has received royalties from Smith \& Nephew Inc (Memphis, TN, USA) in an amount of less than USD 100,000 and has partial ownership in Signal Medical Corp (Marysville, MI, USA) receiving USD 0 during the study period. The institution of one or more of the authors (LAW, MER) has received funding from Signal Medical Corp unrelated to this research. All ICMJE Conflict of Interest Forms for authors and Clinical Orthopaedics and Related Research ${ }^{\mathbb{R}}$ editors and board members are on file with the publication and can be viewed on request.

Clinical Orthopaedics and Related Research ${ }^{\mathbb{R}}$ neither advocates nor endorses the use of any treatment, drug, or device. Readers are encouraged to always seek additional information, including FDAapproval status, of any drug or device prior to clinical use. Each author certifies that his or her institution approved the human protocol for this investigation, that all investigations were conducted in conformity with ethical principles of research, and that informed consent for participation in the study was obtained.

L. A. Whiteside ( $\square)$, M. E. Roy

Missouri Bone and Joint Research Foundation, Missouri Bone and Joint Center, 1000 Des Peres Road, Suite 150, St Louis, MO 63131, USA

e-mail: whiteside@whitesidebio.com infections in well-ingrown cementless stems; and (3) what complications were associated with this approach in a small case series.

Methods Between January 2002 and July 2013, 30 patients (30 hips) presented to the senior author for treatment of infected THA. Of those, 21 patients ( 21 hips) with infected cemented THAs underwent débridement and single-stage revision to cementless total hip implants followed by catheter infusion of intraarticular antibiotics. Nine patients (nine hips) with late-onset acute infections in cementless THA had bone-ingrown implants. These patients were all more than 2 years from their original surgery and had acute symptoms of infection for 4 to 9 days. Seven had their original THA elsewhere, and two were the author's patients. All were symptom-free until the onset of their infection, and none had postoperative wound complications, fever, or prolonged pain suggestive of a more chronic process. They were treated with débridement and head and liner exchange, again followed by catheter infusion of intraarticular antibiotics. During this time period, this represented all infected THAs treated by the senior author, and all were treated with this protocol; no patient underwent two-stage exchange during this time, and no patients were lost to followup. At the time of the surgery, two Hickman catheters were placed in each hip to begin intraarticular delivery of antibiotics in the early postoperative period. Antibiotics were infused daily into the hip for 6 weeks with the tubes used for infusion only. Eleven of the single-stage revisions and four of the hips treated with débridement had methicillinresistant Staphylococcus aureus. Patients were considered free of infection if they had no clinical signs of infection and had a normal C-reactive protein and erythrocyte sedimentation rate at 1 year. Complications were ascertained by chart review. 
Results Twenty of $21(95 \%)$ infections in patients who had single-stage revision for chronically infected cemented THA were apparently free from infection and remained so at a mean followup of 63 months (range, 25-157 months). One case grew Candida albicans in the operative cultures and remained free of signs of infection after rerevision followed by infusion of fluconazole. The nine cementless THAs treated with débridement and head/liner exchange all remained free of signs of infection at a mean followup of 74 months (range, 62-121 months). Few complications were associated with the technique. Four patients had elevated serum levels of vancomycin without renal function changes and two patients had transient blood urea nitrogen/creatinine elevations with normal vancomycin levels that resolved with dosage adjustments. No patient had evidence of permanent renal damage. None of the patients in this study developed a chronic fistula or had significant drainage from the catheter site.

Conclusions Single-stage revision for chronically infected cemented THA and débridement of bone-ingrown cementless THA with late-onset acute infection followed with indwelling catheter antibiotic infusion can result in infection eradication even when resistant organisms are involved. Larger study groups would better assess this technique and prospective comparisons to more traditional one- and two-stage revision techniques for infected THA will likely require multi-institutional approaches. Level of Evidence Level IV, therapeutic study.

\section{Introduction}

An established technique for the treatment of chronic infection in THA is generally considered to consist of two-stage revision with an interim antibiotic-loaded polymethylmethacrylate spacer to deliver antibiotics to the local area combined with intravenous antibiotics to deliver antibiotics systemically [3, 24, 33, 38]. By contrast, singlestage revision procedures performed with or without cement fixation also are used [5, 33, 58], and no clear evidence exists that two-stage revision yields superior results to single-stage protocols $[5,14,18,21,23,33,58]$. Additionally, the interim antibiotic cement spacers associated with two-stage exchange may carry some risk including dislocation of the spacer [15, 26, 43], fracture of the hip [15], fracture of the spacer $[9,15,26]$, migration of the spacer leading to bone damage or even vascular injury [48], rare reports of renal injury from antibiotic elution, and the need to remove the spacer should these complications occur $[1,10,13,37,40,42,52]$.

High local antibiotic concentration around the site of an infected THA is a matter of major clinical importance, because bacteria protected by biofilm require concentrations that are orders of magnitude greater than the minimal inhibitory concentration for the planktonic forms of the same bacterium to eliminate resistant organisms that are protected by the glycocalyx [8, 11, 12, 16, 32]. Intravenous antibiotics generally do not achieve these levels of concentration in synovial fluid, but instead achieve levels around two to three times the minimal inhibitory concentration (MIC) [46]. Antibiotic-impregnated cement spacers achieve local concentrations of vancomycin over $1500 \mu \mathrm{g} /$ $\mathrm{mL}$ (three orders of magnitude greater than the MIC for planktonic forms) during the first few days after surgery, but those levels decrease rapidly during the succeeding days [2, 22]. In contrast, direct antibiotic infusion through a catheter, as first described by Perry et al., achieves extremely high local levels of antibiotics for a prolonged period of time [44, 46, 54-56] and can be discontinued if toxicity or sensitivity occurs [55]. This method has been effective in treating infected TKA in challenging conditions [54-56], and use of a cannulated spacer for delivering antibiotics into an infected total hip after débridement has been reported with successful early results [25]. However, that report described a temporary spacer that required subsequent removal to prepare for the final implant. Once the spacer was removed, no antibiotic infusion remained to provide protection for the final arthroplasty implants.

Single-stage revision arthroplasty combined with local antibiotic infusion has been described fairly extensively for TKA [54-56], but this procedure has not been described in detail for THA. Because two-stage revision involves two major operations, and antibiotic spacers themselves may create complications, a procedure that could obviate both the second stage and the spacers, while producing very high antibiotic concentration that could be continued for weeks, would be advantageous. Intraarticular infusion of antibiotics provides the means of delivering the drug in high concentrations in the presence of a revision arthroplasty, allows the dosage of the antibiotic to be adjusted, and even allows addition of a different antibiotic if necessary.

We describe a protocol for intraarticular delivery of antibiotics to the hip through an indwelling catheter combined with one-stage revision surgery and examine (1) the success as judged by eradication of infection at 1 year when treating chronically infected cemented stems; (2) success at treating patients with late-onset acute infections in well-ingrown cementless stems; and (3) the complications associated with this approach in a small case series.

\section{Patients and Methods}

Data were collected as part of a longitudinally maintained institutional database and analyzed retrospectively on all patients presenting with infected THA treated by the senior author (LAW) between January 2002 and July 2013. A 
total of 30 patients were seen with the diagnosis during this time period. Patients were divided into two groups based on presentation: (1) chronic infection of a THA with a cemented stem; and (2) late-onset acute infection of a wellingrown cementless THA. Chronic infection was defined as infection beginning within the first 3 months after the primary procedure and persisting for more than 6 months. Late acute-onset infection was defined as infection appearing more than 2 years after the primary procedure with signs and symptoms persisting less than 2 weeks before presentation. No patients with loose chronically infected cementless THAs or late-onset acute infection of cemented THAs presented for treatment during this time period. All patients with chronically infected cemented THAs were treated with single-stage revision to cementless implants and intraarticular antibiotic infusion through a catheter. All patients with well-ingrown cementless implants with late-onset acute infection were treated with débridement and femoral head and polyethylene liner exchange combined with intraarticular antibiotic infusion through a catheter. No patient in either group was treated with a different protocol such as two-stage exchange with an antibiotic spacer.

There were 21 patients ( 21 hips; 15 men, six women) in the chronically infected cemented group (Table 1). Five patients (five hips) had infected primary THA, 12 patients (12 hips) had infected revision of primary THA, and four patients (four hips) had reinfection after failed two-stage revision of infected THA. All hips had loose femoral and acetabular components and thus were not candidates for débridement and retention of implants. Comorbidity classification was done for each patient preoperatively by the anesthesiology consultant using the American Society of Anesthesiologists classification system (www.asahq.org/resources/clinicalinformation/asa-physical-status-classification-system) and comorbidities were listed (Table 1) [47]. Additionally, bone quality was assessed on anterior and lateral radiographs. All 21 patients in this group had cortical thinning and widening of the medullary canal distal to the femoral component. Thirteen had moderate osteolysis involving the proximal third of the femur and peripheral areas of the acetabulum. Eight had severe osteolytic lesions involving the proximal femur and acetabular margins. Four had destruction and fragmentation of the greater trochanter. Mean followup of this group was 63 \pm 51 months (range, $25-157$ months). Nineteen of 21 patients were still being followed at the time of this analysis; two patients died of causes unrelated to their hip surgery after their second year of followup and were judged to be free of infection up to the time of death.

The surgical technique in the infected cemented group included removal of all cement and direct exposure of all bone-cement interfaces to allow the underlying bone to be débrided with curettage and burring with a high-speed burr.
Bivalve osteotomy of the femur was done in all hips to achieve complete exposure of the bone-cement interface. Bacteriologic diagnosis was obtained in all hips with 10 cultures (five aerobic, acid-fast bacilli and fungus; five anaerobic) of tissue biopsies taken for each patient at surgery. All patients had two or more positive tissue cultures and thus conformed to the Musculoskeletal Infection Society criteria for infection [41]. Six patients (six hips) had positive cultures for methicillin-resistant Staphylococcus aureus (MRSA), five patients (five hips) had methicillin-resistant Staphylococcus epidermidis (MRSE), five patients (five hips) had methicillin-sensitive $S$ aureus (MSSA), three patients (three hips) had methicillin-sensitive $S$ epidermidis (MSSE), one patient (one hip) had Serratia marcescens that was sensitive to gentamicin, and one patient (one hip) had Candida albicans. All hips had repeated irrigation with hand-pump pulsed lavage with 250,000 units/L polymyxin and $1000 \mathrm{mg} / \mathrm{L}$ vancomycin in normal saline. The rate of irrigation was approximately $1 \mathrm{~L}$ per hour. Antiseptic solution was not used. A fully porouscoated revision stem (Quatroloc; Signal Medical Corp, Marysville, MI, USA) and porous-coated acetabular component with ceramic femoral head (MicroSeal; Signal Medical Corp) were implanted in all hips.

There were nine patients (nine hips; five men, four women) in the late-onset acute infection group. All were well-ingrown cementless primary THAs. The time from the original procedure to symptoms of infection was $87 \pm 23$ months (range, 61-133 months). All patients presented for treatment 4 to 9 days after onset of symptoms. Comorbidities and American Society of Anesthesiologists classification are noted (Table 1). Bone quality assessment revealed no severe osteolysis. All patients had mild proximal femoral stress relief osteopenia. Mean followup duration was 74 months (range, 62-121 months) and no patient was lost to followup during this study. Surgical technique in this group consisted of thorough surgical débridement and head and polyethylene liner exchange. All hips had repeated irrigation with hand-pump pulsed lavage with 250,000 units/L polymyxin and $1000 \mathrm{mg} / \mathrm{L}$ vancomycin in normal saline. The rate of irrigation was approximately $1 \mathrm{~L}$ per hour. Betadine and other antiseptic solutions were not used. Bacteriologic diagnosis was obtained in all hips with 10 cultures of tissue biopsies taken at surgery for each patient. Four patients (four hips) had multiple positive cultures for MRSA and five patients (five hips) had multiple positive cultures for MSSA.

At closure, all infected revision hips in both groups received two Hickman catheters inserted into the intraarticular space (Bard Access Systems, Inc, Salt Lake City, UT, USA), and these catheters were maintained for 6 weeks for direct intraarticular infusion of antibiotics (Fig. 1). Hickman catheters are silicon rubber tubes with a 
Table 1. Patient demographics

\begin{tabular}{|c|c|c|}
\hline THA groups & Chronically infected/cemented THA & Hematogenous infection cementless THA \\
\hline Number of patients & 21 (15 men, 6 women $)$ & 9 (5 men, 4 women) \\
\hline Mean age \pm SD (years) & $67 \pm 7(58-79)$ & $67.1 \pm 3.6($ range, $65-76)$ \\
\hline Mean followup \pm SD (months) & $63 \pm 51($ range, $25-157)$ & $59 \pm 23$ (range, $25-79)$ \\
\hline ASA classification & Number of patients & Number of patients \\
\hline I & 0 & 0 \\
\hline II & 14 & 6 \\
\hline III & 5 & 3 \\
\hline IV & 2 & 0 \\
\hline $\mathrm{V}$ & 0 & 0 \\
\hline \multicolumn{3}{|l|}{ Smoking status } \\
\hline Smoker & 6 & 0 \\
\hline Nonsmoker & 15 & 9 \\
\hline \multicolumn{3}{|l|}{ Comorbidities } \\
\hline Obesity $\left(\mathrm{BMI}>35 \mathrm{~kg} / \mathrm{m}^{2}\right)$ & 7 (3 men, 4 women) & 5 ( 2 men, 3 women $)$ \\
\hline CAD & 17 (11 men, 6 women) & 7 (5 men, 2 women) \\
\hline Type II diabetes & 9 (3 men, 6 women) & 5 (3 men, 2 women) \\
\hline Chronic stasis dermatitis & 8 (3 men, 5 women) & 4 (2 men, 2 women) \\
\hline Chronic renal failure-hemodialysis & 0 & 3 (1 man, 2 women) \\
\hline Peripheral vascular disease & 2 (2 men) & 0 \\
\hline Two comorbidities & 21 & 4 (3 men, 1 woman) \\
\hline Three comorbidities & 21 & 4 (3 men, 1 woman) \\
\hline
\end{tabular}

ASA = American Society of Anesthesiologists; BMI = body mass index; CAD = coronary artery disease.

fibrous cuff that is placed approximately $1 \mathrm{~cm}$ under the skin. The tubes are inserted with a trocar (available in the Hemovac 1/8-inch kit) from outside into the joint (Fig. 2). Before the fibrous cuff is inserted beneath the skin, the tube is marked for the correct position (Fig. 3). Then the tube is pulled so the fibrous cuff is beneath the skin to the depth indicated on the mark on the tube (Fig. 4). The tube is sutured with 2-0 silk sutures (Fig. 5). Once the fibrous cuffs are positioned correctly, the tubes are cut to the correct length to lie safely in the hip (Fig. 6). The fibrous cuff becomes ingrown with fibrous tissue, which seals the interface between the tube and the subcutaneous tissue to prevent external contamination and joint fluid leakage. Both tubes were used for infusion to increase patency and the chances of maintaining an open catheter during treatment. The joint fluid was not drained; instead, the antibiotics were left in the joint to maintain a high local concentration and also to produce a significant serum level (Fig. 7) [46, 54-56]. No concomitant intravenous antibiotics were administered after the intraarticular dose was established.

Infusion of vancomycin was started as soon as the incision was sealed and dry (usually 2 days), and the dose was increased gradually as the incision was monitored. Vancomycin was used because of its effectiveness against bacteria in glycocalyx biofilms. This property of vancomycin makes it preferable to cephalosporins when infusing directly into joints that have metal implants $[12,27]$. Gentamicin was used only in the one patient with a gram-negative bacterium that was sensitive to this antibiotic. The beginning test dose was $100 \mathrm{mg}$ vancomycin in $3 \mathrm{~mL}$ sterile water, and if this was tolerated (denoted by lack of wound drainage or irritation), the dose was increased daily to achieve the final dose of 400 or 500 $\mathrm{mg}$ in 5 or $6 \mathrm{~mL}$ sterile water. Sterile water was used instead of normal saline to help avoid precipitation of the vancomycin and plugging of the tubes. Five to 7 days generally were required to achieve a stable maintenance dose, but if wound drainage occurred, the process required as long as 14 days. Six patients had one catheter become plugged during treatment, but none of the patients lost both catheters. A more dilute solution of vancomycin-500 mg in $10 \mathrm{~mL}$ sterile water-is now the standard vancomycin maintenance dose because of the drug's tendency to precipitate. The starting dose for gentamicin was $10 \mathrm{mg}$ in 3 $\mathrm{mL}$ normal saline, and the maintenance dose was $40 \mathrm{mg}$ in $4 \mathrm{~mL}$ normal saline. The maintenance dose was given once daily, and the catheters were alternated so that they remained patent. The antibiotic solution was prepared by an infusion company and the antibiotic dosage was 


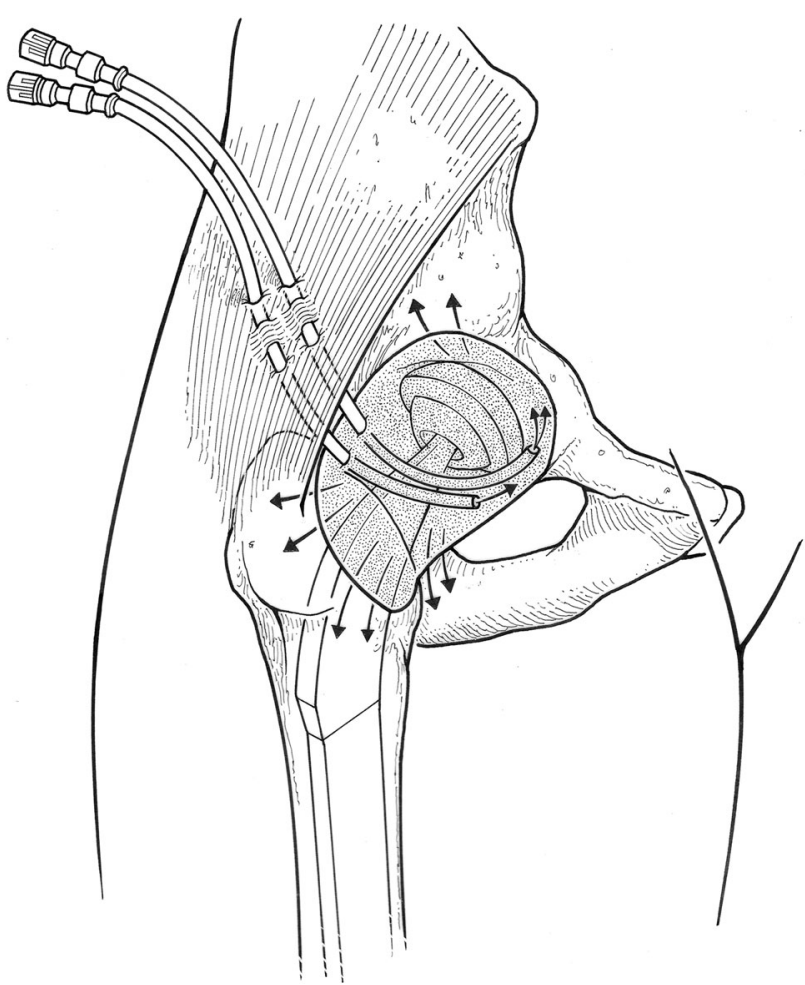

Fig. 1 This drawing illustrates the components and placement of intraarticular infusion catheters used in the hip after débridement or one-stage revision. The injection portals remain outside the skin, the fibrous cuffs are placed approximately $1 \mathrm{~cm}$ under the dermis, and the catheter tubes are placed in the joint and extend under the femoral neck to deliver antibiotics directly into the joint. These catheters are used for infusion only. Aspiration and drainage are not done so that the concentration remains high in the joint cavity and the antibiotics diffuse into the synovial capillaries and lymphatic channels. This route of delivery also achieves a substantial peripheral serum concentration of antibiotics.

administered by a home health nurse or by the patient and family after appropriate training.

Serum antibiotic concentration and renal function studies (blood urea nitrogen [BUN] and creatinine) were tested weekly for vancomycin and twice weekly for gentamicin (Table 2). The doses were adjusted in patients with elevated serum antibiotic levels, and the antibiotics were discontinued in patients whose kidney function values were elevated. After renal function values normalized, antibiotics were resumed at half the previous maintenance dose, and the renal function studies were repeated every 2 days.

After 6 weeks of infusion, the catheters were removed under local anesthesia in the operating room. The fibrous cuff around the Hickman catheters becomes ingrown with dense fibrous tissue, so the catheters must be removed by sharp dissection under sterile conditions. The local area was infiltrated with a solution of $1 \%$ lidocaine with epinephrine and an incision was made above and below the catheters. The fibrous cuff was dissected sharply from the

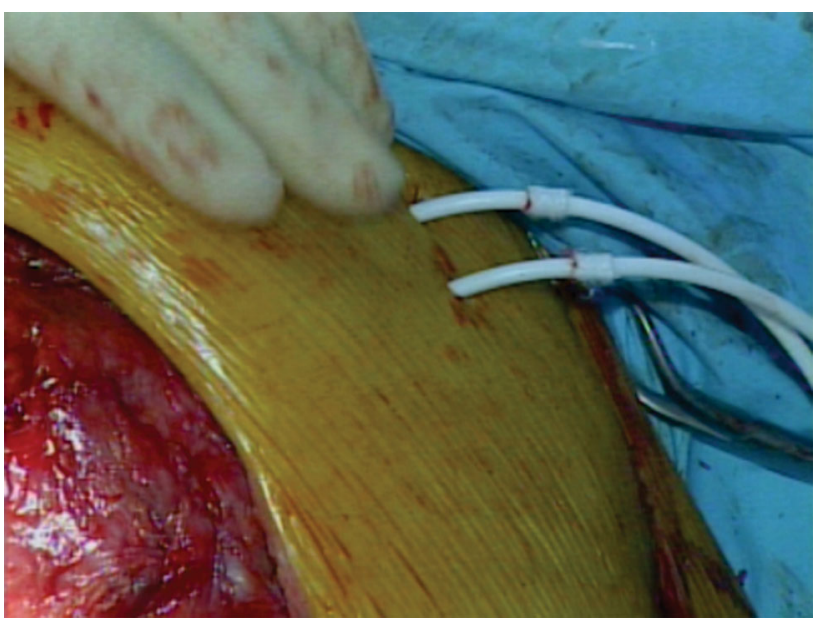

Fig. 2 This intraoperative photograph shows the Hickman catheters just before placement of the fibrous cuff.

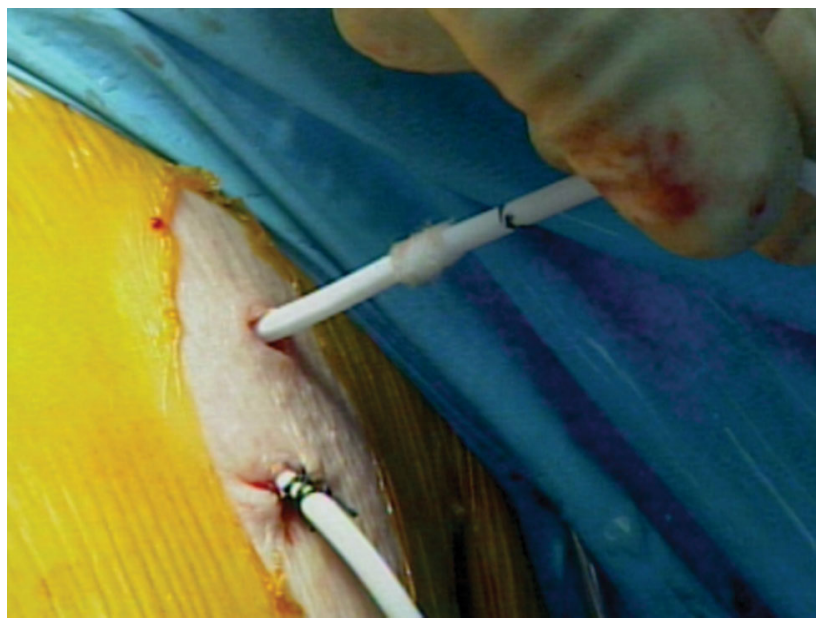

Fig. 3 This intraoperative photograph of both Hickman catheters indicates how the lower tube has been inserted and secured with two sutures. The upper tube has been marked for depth of insertion of the fibrous cuff.

surrounding muscle and fat using a \#15 knife blade (Bard Access Systems, Inc). The catheters are made of soft silicone and are easily transected, so this was done carefully to avoid cutting through one of the tubes and allowing the free end to escape into the joint. Because the tubes lead directly into the hip, special care was taken to close the entry into the tracts left by the tubes. They were oversewn with 2-0 Vicryl sutures until completely sealed; then the subcutaneous tissue was closed tightly with interrupted inverted 20 Vicryl sutures. The skin was closed snugly with interrupted silk sutures.

None of the patients were maintained on oral suppressive antibiotics after discontinuation of intraarticular infusion. 


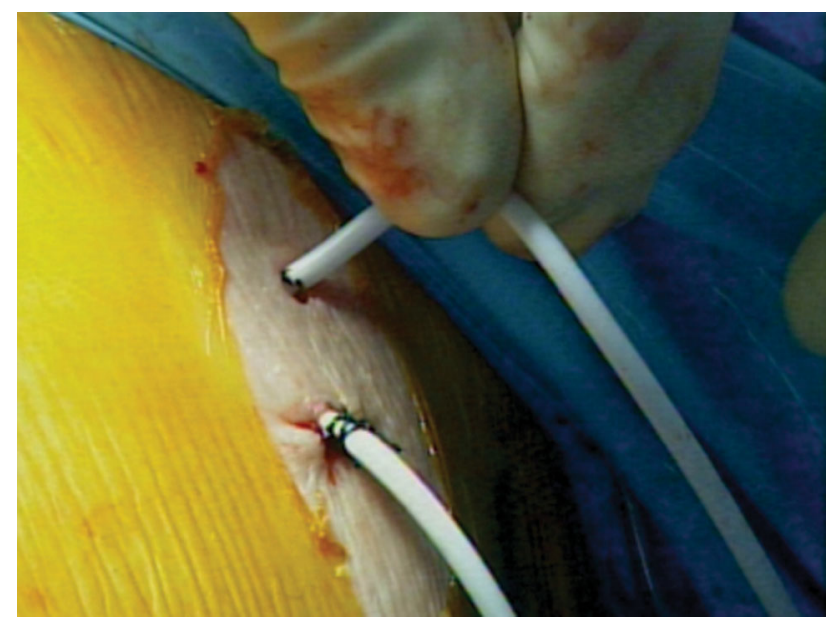

Fig. 4 This intraoperative photograph shows both Hickman catheters with the upper tube pulled through to the depth marked.

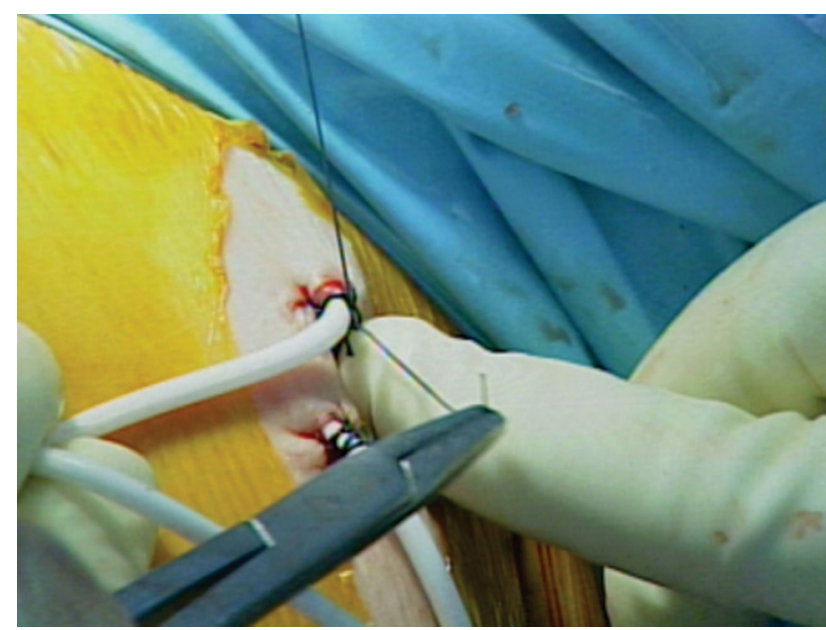

Fig. 5 This intraoperative photograph shows both Hickman catheters, and the upper tube is secured with two 2-0 silk sutures.

The patients were seen at monthly intervals for 3 months and then at yearly intervals permanently. At each visit they were evaluated for clinical signs of recurrent infection including pain, erythema, swelling, induration, and drainage. Patients were asked to rate pain as none, mild, moderate or severe. C-reactive protein (CRP) levels and erythrocyte sedimentation rate (ESR) were determined before surgery and at each followup interval. Successful control of infection was indicated by progressive improvement in all subjective and objective clinical signs, continuous decrease in CRP and ESR during the first year, and normal CRP and ESR after 1 year (Table 2). If the patients were to fail to improve, an extensive workup would be initiated to evaluate for infection with fungus or other atypical organisms or to search for abscesses or foreign material. Complications in this series included

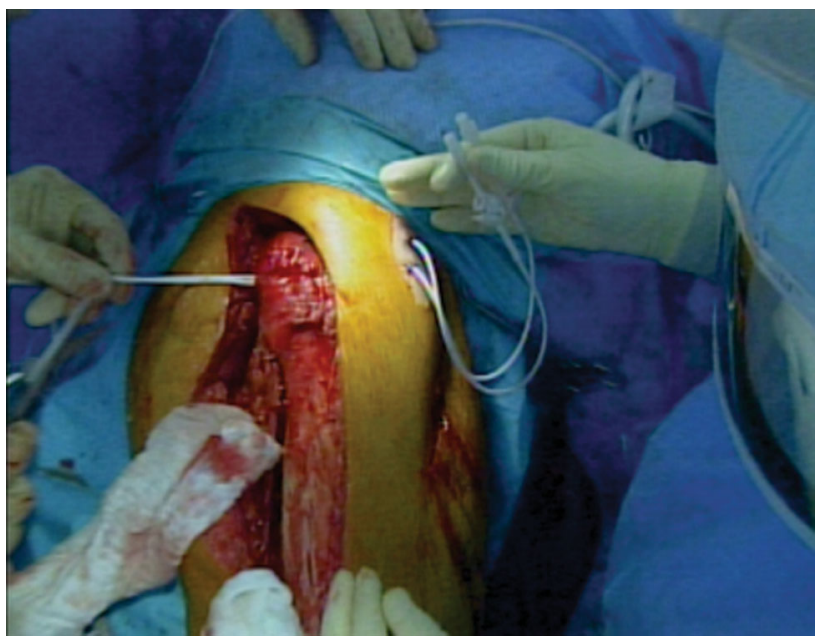

Fig. 6 This intraoperative photograph shows the Hickman catheters exiting the surgical wound. The Hickman catheter tubes will be cut to lie safely within the joint.

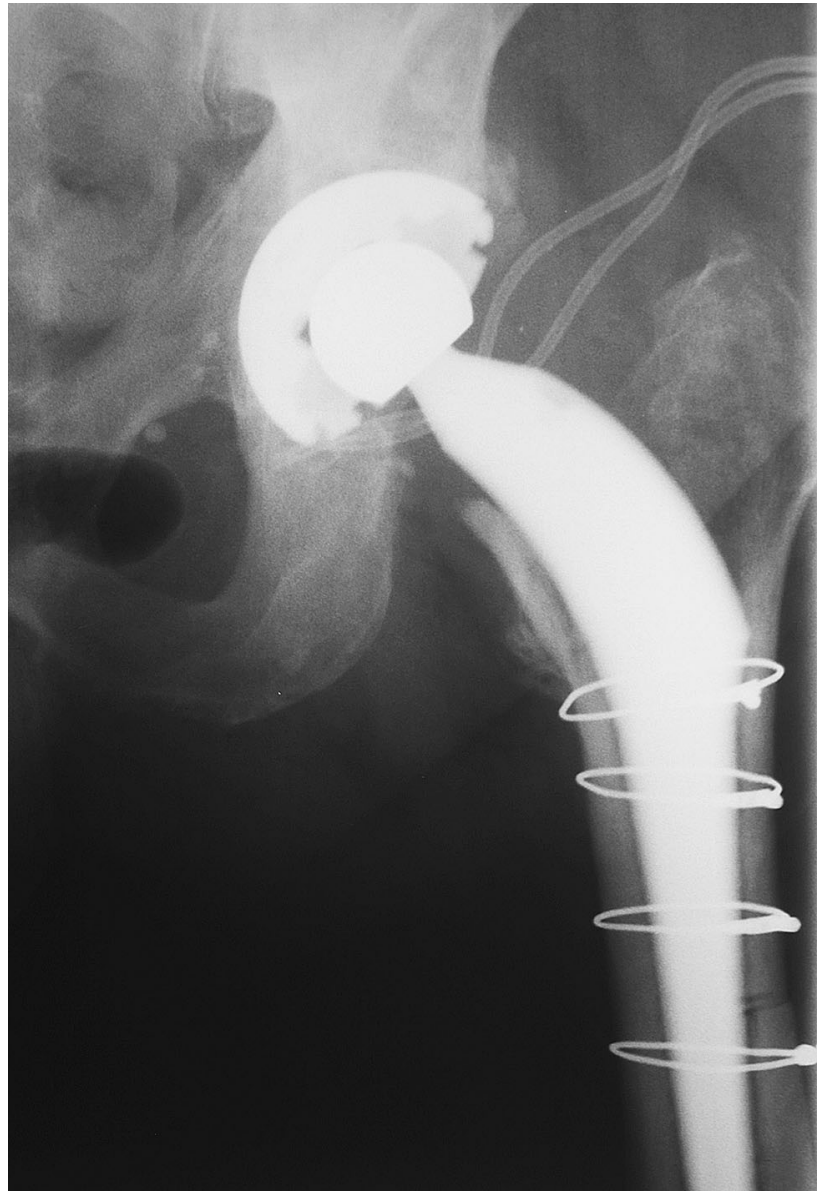

Fig. 7 This radiographic image was taken 1 month postoperatively with infusion catheters in place after single-stage revision.

elevated BUN and creatinine levels, elevated vancomycin serum levels, and clogged catheter tubes and were ascertained by retrospective chart review. 
Radiographic evaluation was done by one of the authors (LAW) immediately after surgery and at each office visit thereafter. AP and lateral views of the hip were evaluated for signs of failure of bone ingrowth to the femoral stem and acetabular component. Signs of failure of bone ingrowth of the acetabular component in this study included progressive increase in width or length of a radiolucent line measured with a ruler with $1-\mathrm{mm}$ graduation, progressive migration proximally relative to a fixed landmark such as the inferior edge of the teardrop, or progressive change in the abduction angle of the metal shell relative to the transischial line. Progressive movement was defined as measurable movement in the same direction seen on two successive examinations.

Signs of loosening of the femoral component included complete radiolucency around the porous stem, progressive lateral translation of the distal tip, or progressive distal migration of the stem relative to a fixed landmark on the femur such as the proximal edge of the femoral shaft.

Signs of bone ingrowth to the femoral stem included trabecular bone immediately apposed to the surface of the porous stem without radiolucencies separating bone from metal and with bridging of the trabecular lines to adjacent cortical bone. Direct apposition of cortical bone to the distal third of the porous metal surface of the stem with accompanying hypertrophy of the cortical bone visible on sequential radiographs and cortical hypertrophy around the distal half of the stem accompanied by proximal atrophy of bone adjacent to the stem were also considered to be signs of stable osteointegration of the stem.

Synovial fluid concentration of antibiotic levels was not measured in this study, but based on a previously reported infected TKA cohort [46], fluid vancomycin levels well over $4000 \mu \mathrm{g} / \mathrm{mL}$ might be expected in the joint fluid.

\section{Results}

Twenty of the 21 (95\%) single-stage revision hips for chronic infection in cemented stems using this protocol were free of infection based on clinical parameters and serologic measures (CRP and ESR) at 1 year (Table 2) and all remained free of signs and symptoms of infection at a mean followup of 63 months (range, 25-157 months). At 1 year, 17 were free of pain and four reported mild pain. At final followup, 18 reported no pain and three reported mild pain. The patient with the $C$ albicans infection continued to drain postoperatively, and 8 days after surgery, three of five tissue biopsies were read as positive for fungus, although preoperative aspiration of the hip did not reveal this pathogen. The hip was redébrided and revised 11 days after the first revision and started on intraarticular, intravenous, and oral fluconazole. The wound drainage did not 
recur, and the patient is presently 49 months postoperative with no clinical signs of recurrent infection. Two acetabular components migrated proximally $2 \mathrm{~mm}$ between the first month and third month examinations, but both stabilized and neither component tilted or developed progressive radiolucent lines. Two femoral components migrated $2 \mathrm{~mm}$ distally between the first and third month examinations, and two migrated $3 \mathrm{~mm}$ during the same time period. All implants then stabilized, and none has developed other signs of loosening.

All patients had at least two radiographic signs of osteointegration of the femoral component at 3 years postoperatively.

All nine patients (nine hips) who had one-stage débridement and retention of the implants in the setting of late acute-onset infection in well-ingrown cementless stems using this protocol resolved their infection based on clinical parameters and serologic measures (Table 2; CRP and ESR) at 1 year, and all remained free of infection at a mean followup of 74 months (range, 62-121 months). At 1 year and at latest followup, all patients in this group reported no pain.

Few complications were associated with the technique. Four patients had elevated serum levels of vancomycin and their dosages were decreased to $250 \mathrm{mg}$ daily but none had elevated BUN or creatinine levels. Two patients had elevated BUN and creatinine levels but had nontoxic levels of vancomycin. Vancomycin was discontinued in both patients and restarted at a dose of $250 \mathrm{mg}$ daily 1 week later after the renal function studies normalized. No patient had evidence of permanent renal damage. None of the patients in this study developed a chronic fistula or had significant drainage from the catheter site.

\section{Discussion}

Treatment of infected THA remains challenging with substantial patient morbidity and expense associated with traditional two-stage exchange requiring interim antibiotic spacer and intravenous antibiotic management. Although one-stage exchange has shown promise [5, 18, 21, 23, 33], the efficacy of this treatment in cases of chronic infection and resistant organisms and the need for long-term intravenous antibiotic management may limit its use to selected cases. Treatment of both chronically infected cemented THA and late-onset acute infections in well-ingrown cementless THA using a single-stage revision combined with direct catheter infusion of antibiotics into the joint was successful in this small series, both among patients with antibiotic-sensitive and -resistant organisms; no severe complications were attributable to the use the intraarticular antibiotic infusion.
There are numerous limitations of this study, including the small study size, the lack of a control or comparative group, and the fact that it reflects the results of a single surgeon. However, the length and completeness of followup and the high proportion of patients with successful treatment all illustrate that the protocol may be effective for managing a difficult group of patients. In addition, the nature and size of the study precluded multivariate analysis, but the group was not preselected and patients with a variety of comorbidities, organisms, and with varying bone quality were managed successfully. Finally, nearly all the hips in this study were infected with staphylococcus, so little can be determined regarding the effectiveness of this method of treatment for Gram-negative, mixed bacterial, or fungal infections. However, staphylococcal infections, especially those involving methicillin-resistant bacteria, are difficult to treat $[28,34,59]$ and certainly represent a challenging test for our method.

Twenty of the 21 (95\%) single-stage revision procedures for chronic infection in patients with cemented stems using this protocol were successful, the single exception being a case of fungal infection that was only diagnosed by intraoperative cultures. Although cemented revision of infected THA using a two-stage protocol with an interim antibiotic spacer is still standard practice [3, 24, 33, 38], both twostage revision with cementless implants and single-stage revision surgery commonly are reported to be highly successful [5, 18, 23, 33, 58]. Two-stage cementless revision has been reported to eliminate signs of infection in $95 \%$ of cases [6], and cementless two-stage revision followed by rerevision is reported to achieve control of infection in $98 \%$ of cases [29]. No apparent advantage is conferred by using antibiotic-loaded cement in the second stage [35].

Cementless single-stage revision for infection was successful in $98 \%$ of cases when combined with antibioticinfused allograft [57], yet others have reported a much lower success rate of $76 \%$ with the single-stage technique [21]. In addition, reported results with resistant organisms seem to indicate that success rates decline when standard methods of antibiotic delivery are used for treatment of these types of bacteria. One very experienced group reports equivalent success with cemented and cementless revision for infected THA with approximately $10 \%$ developing recurrent infection when treating antibiotic sensitive organisms (MSSA and MSSE), whereas $21 \%$ of the cases with antibiotic resistant organisms (MRSA and MRSE) developed recurrent infection. Other studies have noted recurrent infection occurring in $21 \%-48 \%$ of hips infected with MRSA or MRSE [28, 34] with standard spacer and intravenous antibiotic techniques. In contrast, the results reported in this study may suggest that the antibiotic delivery system offered an advantage in treating THA infected with resistant organisms, possibly as a result of the 
effect of prolonged exposure to local antibiotics in high concentration on biofilm-forming bacteria protected by glycocalyx.

All nine of the patients with infection in the setting of well-ingrown cementless stems resolved their infection with thorough débridement, head and liner exchange, and intraarticular infusion.

These results are in distinct contrast to other reports of treatment of infected THA with retention of implants. Zurcher-Pfund et al. [59] found that $33 \%$ of patients treated with débridement and retention of their implants survived without reinfection if the patients had antibiotic-sensitive bacteria, but that no patient who had either MRSA or MRSE was treated successfully. A similar study [30] reported that $96 \%$ of patients treated with débridement and implant retention had successful treatment and avoided reinfection, but that only $45 \%$ were successfully treated if the infecting organism was staphylococcus. Reports of revision of cementless THA with late-onset acute infection are scarce in the literature. One study reports five of six late hematogenous infections in ingrown cementless THAs successfully treated with débridement, gentamicin fleece insertion, and intravenous antibiotics [19], but other studies report reinfection in $59 \%$ of acute hematogenous staphylococcal infections where the prosthesis was retained [53]. Given the results in our study, one might speculate that the depot antibiotic technique of inserting gentamicin fleece around the implants before closure may confer a similar benefit to the local antibiotic installation method.

Our approach demonstrated few complications. Some concerns have been raised about formation of sinus tracts with techniques that use indwelling catheters. Fistulas are more likely to occur when nonsealed catheters are used, which allow epithelialization of the tract with epidermal tissue. Hickman catheters are sealed with a fibrous cuff that is located a centimeter below the skin. Although the tracts may have epithelialized from the skin down to the cuff during treatment, this outer portion of the tracts and the fibrous cuffs are excised when the catheters were removed. The entrances into the deeper portion of the tract, which does not have an epidermal lining, were closed with sutures before two-layer closure of the subcutaneous tissue. We did not observe any fistula or sinus tract formation in this series, and none has been reported with treatment of infected THAs and TKAs using this technique over the past 15 years $[46,54,55]$. This may be because the entire area is in an active phase of healing when the catheters are removed, and the catheter tract heals rapidly. Meticulous closure also likely plays an important role in successful use of this technique. Similarly, we did not observe significant issues with renal toxicity related to our infusion technique of antibiotics, typically vancomycin, and the cases with elevated serum antibiotic levels or evidence of renal toxicity were managed easily with cessation of intraarticular antibiotic infusion until serum levels and renal function returned to normal. This is in contrast to cases with large antibiotic spacers and other depot techniques that require reoperation and extirpation of the depot device in elderly debilitated patients [42].

The use of this method of intraarticular infusion of bactericidal antibiotics has also been shown effective in controlling infection in TKA cases growing methicillinresistant Staphylococcus species $[55,56]$ and also in difficult cases of rerevision for infection of total knees after failed two-stage revision surgery [54]. Vancomycin concentrations well over $4000 \mu \mathrm{g} / \mathrm{mL}$ are readily obtained in the knee immediately after infusion, and with a daily infusion regimen, this level can be achieved daily for 6 weeks or longer [46]. Moderate concentrations of antibiotics are necessary to prevent biofilm formation [11, 12], but once biofilm and persister cells have formed, concentrations of orders of magnitude greater (as high as $4000 \mu \mathrm{g}$ / $\mathrm{mL}$ ) are necessary to eradicate the bacteria within the biofilm [7, 8, 11, 12, 17, 27, 31, 45, 49, 50]. Clearly, some risk of toxicity exists with direct intraarticular infusion of antibiotics, but this method may be the safest way to achieve high antibiotic levels over time [54-56] while allowing for the opportunity to decrease the dose, change antibiotics, or stop them altogether in the case of allergic reactions or renal toxicity. Depot methods can certainly achieve high levels during the early postoperative period, but their effectiveness deteriorates within 3 days [2, 51] and may not achieve levels high enough over time to treat biofilm-dwelling bacteria [22]. In one report, 18 of 20 cement spacers cultured using sophisticated techniques at the time of spacer removal and final reimplantation grew resistant organisms [39]. Finally, depot methods for antibiotic delivery require extensive surgery for removal of the depot materials in cases with toxic effects of the antibiotics [36].

Single-stage revision of infected THA is gaining wider acceptance [5, 33, 58], and antibiotic-impregnated bone cement is not routinely used during the reimplantation phase of infected THA [4, 6, 20, 29, 33, 35]. With the addition of antibiotics infused directly into the joint, revision of these difficult cases, even when infected with resistant organisms, may have a high likelihood of successfully eradicating the infection and eliminating the morbidity of a second procedure. Still, two-stage revision is indicated in certain cases, even when this technique of intraarticular infusion is used. Although no such cases were performed during the study period, recent experience in our clinic has included cases with abscesses, scarred subcutaneous tissue, and deficient soft tissue coverage that precluded secure closure. In our opinion, these cases are not good candidates for one-stage reimplantation, and 
patients in this setting functioned with a resection arthroplasty until the wound was well enough healed to accept hip reimplantation.

Although this technique of intraarticular infusion can be laborious for the nursing management team postoperatively, we feel there are stated advantages of achieving a much higher local concentration of antibiotics to assist in eradicating bacteria and biofilms. Further efforts to study this technique in larger comparative series with other established methods of one- and two-stage exchange for this difficult problem are warranted.

Acknowledgments We thank William C. Andrea CMI, for preparation of the illustration, and Diane J. Morton MS, for editorial assistance with the manuscript.

\section{References}

1. Aeng ES, Shalansky KF, Lau TT, Zalunardo N, Li G, Bowie WR, Duncan CP. Acute kidney injury with tobramycin-impregnated bone cement spacers in prosthetic joint infections. Ann Pharmacother. 2015;49:1207-1213.

2. Anguita-Alonso P, Rouse MS, Piper KE, Jacofsky DJ, Osmon DR, Patel R. Comparative study of antimicrobial release kinetics from polymethylmethacrylate. Clin Orthop Relat Res. 2006;445:239-244.

3. Berend KR, Lombardi AV Jr, Morris MJ, Bergeson AG, Adams JB, Sneller MA. Two-stage treatment of hip periprosthetic joint infection is associated with a high rate of infection control but high mortality. Clin Orthop Relat Res. 2013;471:510-518.

4. Buttaro MA. Bone grafting and two-stage revision total hip arthroplasty. Hip Int. 2012;22(Suppl 8):S69-S74.

5. Callaghan JJ, Katz RP, Johnson RC. One-stage revision surgery of the infected hip. A minimum 10-year followup study. Clin Orthop Relat Res. 1999;369:139-143.

6. Camurcu Y, Sofu H, Buyuk AF, Gursu S, Kaygusuz MA, Sahin V. Two-stage cementless revision total hip arthroplasty for infected primary hip arthroplasties. J Arthroplasty. 2015;30: $1597-1601$.

7. Cerca N, Martins S, Cerca F, Jefferson KK, Pier GB, Oliveira R, Azeredo J. Comparative assessment of antibiotic susceptibility of coagulase-negative staphylococci in biofilm versus planktonic culture as assessed by bacterial enumeration or rapid XTT colorimetry. J Antimicrob Chemother. 2005;56:331-336.

8. Cha JO, Park YK, Lee YS, Chung GT. In vitro biofilm formation and bactericidal activities of methicillin-resistant Staphylococcus aureus clones prevalent in Korea. Diagn Microbiol Infect Dis. 2011;70:112-118.

9. Citak M, Masri BA, Springer B, Argenson JN, Kendoff DO. Are preformed articulating spacers superior to surgeon-made articulating spacers in the treatment of PJI in THA? A literature review. Open Orthop J. 2015;9:255-261.

10. Curtis JM, Sternhagen V, Batts D. Acute renal failure after placement of tobramycin-impregnated bone cement in an infected total knee arthroplasty. Pharmacotherapy. 2005;25:876-880.

11. Dosler S, Karaaslan E. Inhibition and destruction of Pseudomonas aeruginosa biofilms by antibiotics and antimicrobial peptides. Peptides. 2014;62:32-37.

12. Dosler S, Mataraci E. In vitro pharmacokinetics of antimicrobial cationic peptides alone and in combination with antibiotics against methicillin resistant Staphylococcus aureus biofilms. Peptides. 2013;49:53-58.
13. Dovas S, Liakopoulos V, Papatheodorou L, Chronopoulou I, Papavasiliou V, Atmatzidis E, Giannopoulou M, Eleftheriadis T, Simopoulou T, Karachalios T, Stefanidis I. Acute renal failure after antibiotic-impregnated bone cement treatment of an infected total knee arthroplasty. Clin Nephrol. 2008;69:207-212.

14. Faroug R, Shah Y, McCarthy MJ, Halawa M. Two stage one component revision in infected total hip replacements-two case reports and literature review. Hip Int. 2009;19:292-298.

15. Faschingbauer M, Reichel H, Bieger R, Kappe T. Mechanical complications with one hundred and thirty-eight (antibioticladen) cement spacers in the treatment of periprosthetic infection after total hip arthroplasty. Int Orthop. 2015;39:989-994.

16. Fujiwara S, Miyake Y, Usui T, Suginaka H. Effect of adherence on antimicrobial susceptibility of Pseudomonas aeruginosa, Serratia marcescens, and Proteus mirabilis. Hiroshima J Med Sci. 1998;47:1-5.

17. Gaidhani SV, Raskar AV, Poddar S, Gosavi S, Sahu PK, Pardesi KR, Bhide SV, Chopade BA. Time dependent enhanced resistance against antibiotics and metal salts by planktonic and biofilm form of Acinetobacter haemolyticus MMC 8 clinical isolate. Indian J Med Res. 2014;140:665-671.

18. Gehrke T, Kendoff D. Peri-prosthetic hip infections: in favour of one-stage. Hip Int. 2012;22(Suppl 8):S40-S45.

19. Geurts JA, Janssen DM, Kessels AG, Walenkamp GH. Good results in postoperative and hematogenous deep infections of 89 stable total hip and knee replacements with retention of prosthesis and local antibiotics. Acta Orthop. 2013;84:509-516.

20. Haddad FS, Muirhead-Allwood SK, Manktelow AR, BacareseHamilton I. Two-stage uncemented revision hip arthroplasty for infection. J Bone Joint Surg Br. 2000;82:689-694.

21. Hansen E, Tetreault M, Zmistowski B, Della Valle CJ, Parvizi J, Haddad FS, Hozack WJ. Outcome of one-stage cementless exchange for acute postoperative periprosthetic hip infection. Clin Orthop Relat Res. 2013;471:3214-3222.

22. Hseih PH, Chang YH, Chen SH, Ueng SW, Shih CH. High concentration and bioactivity of vancomycin and aztreonam eluted from Simplex cement spacers in two-stage revision of infected hip implants: a study of 46 patients at an average followup of 107 days. J Orthop Res. 2006;24:1615-1621.

23. Ilchnann T, Zimmerli W, Ochsner PE, Kessler B, Zwicky L, Graber P, Clauss M. One-stage revision of infected hip arthroplasty: outcome of 39 consecutive hips. Int Orthop. 2016; 40: 913-918

24. Jackson WO, Schmalzried TP. Limited role of direct exchange arthroplasty in the treatment of infected total hip replacements. Clin Orthop Relat Res. 2000;381:101-105.

25. Jahoda D, Sosna A, Landor I, Vavrik P, Pokorny D. A cannulated articulating spacer-a functional implant for treatment of infected hip joint prostheses. Acta Chir Orthop Traumatol Cech. 2004;72:73-79.

26. Jung J, Schmid NV, Kelm J, Schmitt E, Anagnostakos K. Complications after spacer implantation in the treatment of hip joint infections. Int J Med Sci. 2009;6:265-273.

27. Keren I, Kaldalu N, Spoering A, Wang Y, Lewis K. Persister cells and tolerance to antimicrobials. FEMS Microbiol Lett. 2004;230:13-18.

28. Kilgus DJ, Howe DJ, Strang A. Results of periprosthetic hip and knee infections caused by resistant bacteria. Clin Orthop Relat Res. 2002;404:116-124.

29. Kim YH, Kim JS, Park JW, Joo JH. Cementless revision for infected total hip replacements. $J$ Bone Joint Surg Br. 2011;93:19-26.

30. Konigsburg BS, Della Valle CJ, Ting NT, Qiu F, Sporer SM. Acute hematogenous infection following total hip and knee arthroplasty. J Arthroplasty. 2014;29:469-472. 
31. Kostenko V, Ceri H, Martinuzzi RJ. Increased tolerance of Staphylococcus aureus to vancomycin in viscous media. FEMS Immunol Med Microbiol. 2007;51:277-288.

32. Lechner S, Lewis K, Bertram R. Staphylococcus aureus persisters tolerant to bactericidal antibiotics. J Mol Microbiol Biotechnol. 2012;22:235-244.

33. Leonard HA, Liddle AD, Burke O, Murray DW, Pandit H. Single- or two-stage revision for infected total hip arthroplasty? A systematic review of the literature. Clin Orthop Relat Res. 2014;472:1036-1042.

34. Leung F, Richards CJ, Garbuz DS, Masri BA, Duncan CP. Twostage total hip arthroplasty: how often does it control methicillinresistant infection? Clin Orthop Relat Res. 2011;469:1009-1015.

35. Masri BA, Panagiotopoulos KP, Greidanus NV, Garbuz DS, Duncan CP. Cementless two-stage exchange arthroplasty for infection after total hip arthroplasty. $J$ Arthroplasty. 2007;22:7278.

36. McLaren AC. Alternative materials to acrylic bone cement for delivery of depot antibiotics in orthopaedic infections. Clin Orthop Relat Res. 2004;427:101-106.

37. Menge TJ, Koethe JR, Jenkins CA, Wright PW, Shinar AA, Miller GG, Holt GE. Acute kidney injury after placement of an antibiotic-impregnated cement spacer during revision total knee arthroplasty. J Arthroplasty. 2012;27:1221-1227.

38. Mortazavi SM, O’Neil JT, Zmistowski B, Parvizi J, Purtill JJ. Repeat 2-stage exchange for infected total hip arthroplasty: a viable option? J Arthroplasty. 2012;27:923-926.

39. Neut D, van de Belt H, Stokroos I, van Horn JR, van der Mei HC, Busscher HJ. Biomaterial-associated infection of gentamicinloaded PMMA beads in orthopaedic revision surgery. J Antimicrob Chemother. 2001;47:885-891.

40. Noto MJ, Koethe JR, Miller G, Wright PW. Detectable serum tobramycin levels in patients with renal dysfunction and recent placement of antibiotic-impregnated cement knee or hip spacers. Clin Infect Dis. 2014;58:1783-1784.

41. Parvizi J, Zmistowski BS, Berbari EF, Bauer TW, Springer BD, Della Valle CJ, Garvin KL, Mont MA, Wongworawat MD, Zalavras CG. New definition for periprosthetic joint infection. Clin Orthop Relat Res. 2011;469:2992-2994.

42. Patrick BN, Rivey MP, Allington DR. Acute renal failure associated with vancomycin- and tobramycin-laden cement in total hip arthroplasty. Ann Pharmacother. 2006;40:2037-2042.

43. Pattyn C, DeGeest T, Ackerman P, Audenaert E. Preformed gentamicin spacers in two-stage revision hip arthroplasty: functional results and complications. Int Orthop. 2011;35:1471-1476.

44. Perry CR, Hulsey RE, Mann FA, Miller GA, Pearson RL. Treatment of acutely infected arthroplasties with incision, drainage, and local antibiotics delivered via an implantable pump. Clin Orthop Relat Res. 1992;281:216-223.

45. Rose WE, Poppens PT. Impact of biofilm on the in vitro activity of vancomycin alone and in combination with tigecycline and rifampicin against Staphylococus aureus. J Antimicrob Chemother. 2009;63:485-488.

46. Roy ME, Peppers MP, Whiteside, LA, LaZear RM. Vancomycin concentration in synovial fluid: direction injection into the knee $\mathrm{v}$ intravenous infusion. J Arthroplasty. 2014;29:564-568.

47. Saklad M. Grading of patients for surgical procedures. Anesthesiology. 1941;2:281-284.

48. Shen DL, Yu CT, Chang IL, Chen SJ. Iliac vessel injury by a cement spacer: report of a case. Int Surg. 2007;92:78-81.

49. Singh R, Ray P, Das A, Sharma M. Role of persisters and smallcolony variants in antibiotic resistance of planktonic and biofilmassociated Staphylococcus aureus: an in vitro study. J Med Microbiol. 2009;58:1067-1073.

50. Smith K, Perez A, Ramage G, Gemmell CG, Lang S. Comparison of biofilm-associated cell survival following in vitro exposure of methicillin-resistant Staphylococcus aureus biofilms to the antibiotics clindamycin, daptomycin, linezolid, tigecycline, and vancomycin. Int J Antimicrob Agents. 2009;33:374-378.

51. van de Belt $H$, Neut D, Schenk W, van Horn JR, van der Mei HC, Busscher JH. Gentamicin release from polymethylmethacrylate bone cements and Staphylococcus aureus biofilm formation. Acta Orthop Scand. 2000;71:625-629.

52. Van Raaij TM, Visser LE, Vulto AG, Verhaar JA. Acute renal failure after local gentamicin treatment in an infected total knee arthroplasty. J Arthroplasty. 2002;17:948-950.

53. Vilchez F, Martinez-Pastor JC, Garcia-Ramiro S, Bori G, Tornero E, Garcia E, Mensa J, Soriano A. Efficacy of débridement in hematogenous and early post-surgical prosthetic joint infections. Int J Artif Organs. 2011;34:863-869.

54. Whiteside LA, Nayfeh TA, LaZear R, Roy ME. Reinfected revised TKA resolves with an aggressive protocol and antibiotic infusion. Clin Orthop Relat Res. 2012;470:236-243.

55. Whiteside LA, Peppers M, Nayfeh TA, Roy ME. Methicillinresistant Staphylococcus aureus in TKA treated with revision and direct intra-articular antibiotic infusion. Clin Orthop Relat Res. 2011;269:26-33.

56. Whiteside LA, Roy ME, Nayfeh TA. Intra-articular infusion: a direct approach to treatment of infected total knee arthroplasty. Bone Joint J. 2016;98(Suppl A):31-36.

57. Winkler H. Bone grafting and one-stage revision of THR-biological reconstruction and effective antimicrobial treatment using antibiotic impregnated allograft bone. Hip Int. 2012;22(Suppl 8):S62-S68.

58. Wroblewski BM. One-stage revision of infected cemented total hip arthroplasty. Clin Orthop Relat Res. 1986;211:103-107.

59. Zurcher-Pfund L, Uckay I, Legout L, Gamulin A, Vaudaux P, Peter R. Pathogen-driven decision for implant retention in the management of infected total knee prostheses. Int Orthop. 2013;37:1471-1475. 